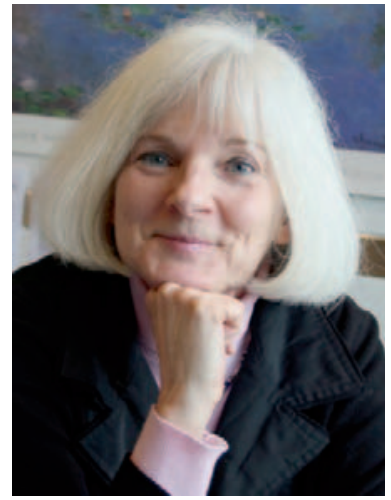

Dr. Barbara Brauckmann, Zürich, Schweiz

\section{Aus unscheinbaren Schränken in eine Museumssammlung: Bücherschätze aus fünf Jahrhunderten}

Anfang 2009 wurden in einem Seminarraum des Institutes für Pharmazeutische Wissenschaften der Eidgenössischen Technischen Hochschule (ETH) Zürich vier schlichte Schränke geöffnet und darin rund 800 alte Bücher mit Ledereinbänden bis hin zu einfachen Pappumschlägen vorgefunden.

Die Abklärung mit verschiedenen Kuratoren machte rasch deutlich, dass diese aus dem Nachlass von Prof. Carl Hartwich (1851-1917) stammenden Schriften für eine derartige Lagerung zu kostbar sind, eine Unterbringung in einem wenig zugänglichen Tresor aber ebenso unbefriedigend wäre.

Da die Bücher als Einheit den Corpus einer typischen Gelehrtenbibliothek aus der Wende des 19. ins 20. Jahrhunderts darstellen, wurde als geeignete Lösung diskutiert, diese zusammen mit seiner umfangreichen pharmakognostischen Sammlung kabinettartig im Pharmazie-Historischen Museum in Basel zu präsentieren. Nach verschiedenen Abwägungen schloss die ETH Zürich mit der Universität Basel einen Vertrag über eine Dauerleihgabe ab. Vor der Unterbringung der Bücher in den Räumlichkeiten am Totengässlein in Basel wurden Umschlag, Titelseiten sowie einige beispielhafte Innenseiten jedes Buches fotografiert und die Fotos in elektronischen Ordnern archiviert.

Am 4. Februar 2011 wurde ein Teil der Bücher den Museumsbesuchern des Pharmazie-Historischen Museums zum ersten Mal ausgestellt.

\section{KARGER}

() 2015 S. Karger GmbH, Freiburg

\section{Publikationen für eine Impression des spannenden Lesestoffs}

Im von Prof. Robert Eder (1885-1944) verfassten Nachruf zum Leben und zur Forschung Hartwichs mit der Auflistung der zahlreichen Publikationen wird deutlich, welch grosse Rolle Gewürze und Genussmittel, aber auch die Mikroskopie im Leben des Pharmazieprofessors spielten [1]. Daher wurden von Martin Kluge, Pharmazie-Historisches Museum der Universität Basel, sowie der im Departement Chemie und Angewandte Biowissenschaften der ETH Zürich tätigen Autorin dieses Beitrags insgesamt acht Artikel über entsprechend ausgewählte Bücher angefertigt und dabei verschiedene Zusammenhänge aufgedeckt und beleuchtet [2-9].

Die beiden Verfasser haben sich dafür virtuell mit Kaufleuten, Botanikern und Ärzten früherer Jahrhunderte auf Reisen in den Orient begeben, begleiteten Gelehrte bei der Erforschung einsamer Bergregionen, besuchten berühmte Universitätsstädte der Renaissance und staunten beim Lesen der sorgfältig und teilweise umständlich formulierten Schilderungen von Land, Menschen und Vegetation. Manchmal fiel auf, wie einschmeichelnd ein Autor, der aus finanziellen Gründen auf die Unterstützung seines Landesherrn angewiesen war, diesem eine ganze Seite mit Lobpreisungen widmete.

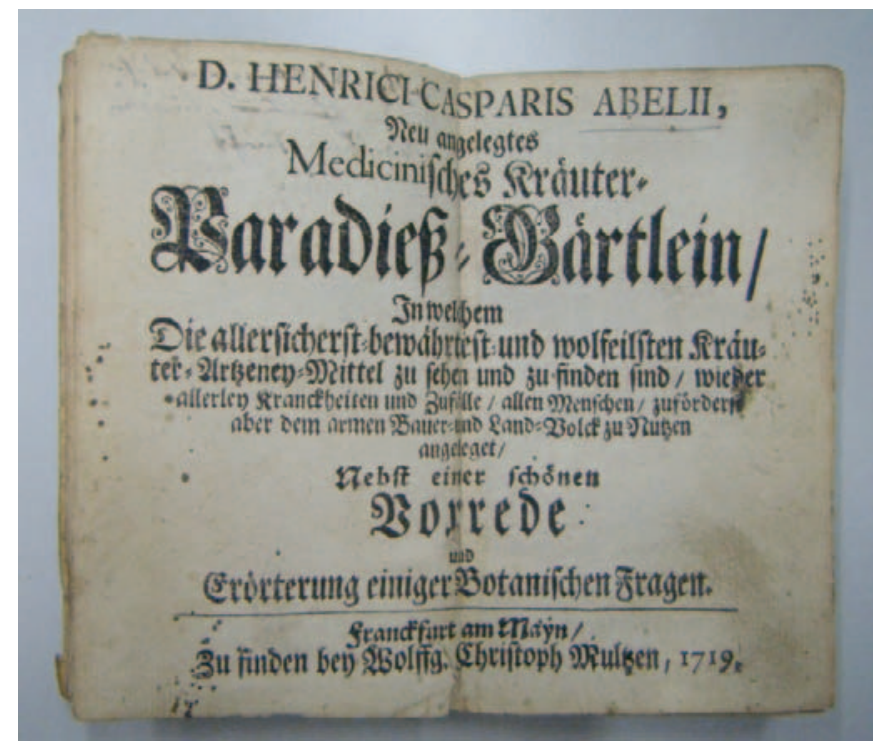

Abb. 1. D. Henrici Casparis Abelii: «Neu angelegtes medicinisches KräuterParadies-Gärtlein, in welchem die allersicherst-bewährtest und wohlfeilsten Kräuter-Artzeney-Mittel zu sehen und zu finden sind». Wolffg. Christoph Multzen, Franckfurt am Mayn, 1719 (Foto: D-CHAB, ETH Zürich). 


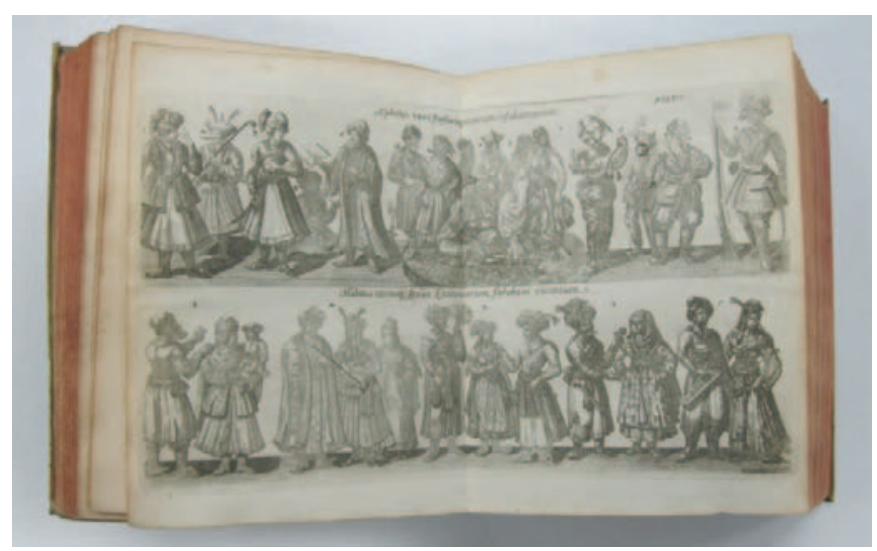

Abb. 2. Engelberto D. Kaempfero: «Amoenitatum Exoticarum politicophysico-medicarum, Fasciculi V, ...». Henrici Wilhelmi Meyeri, Lemgoviae (Lemgo), 1712 (Foto: D-CHAB, ETH Zürich).

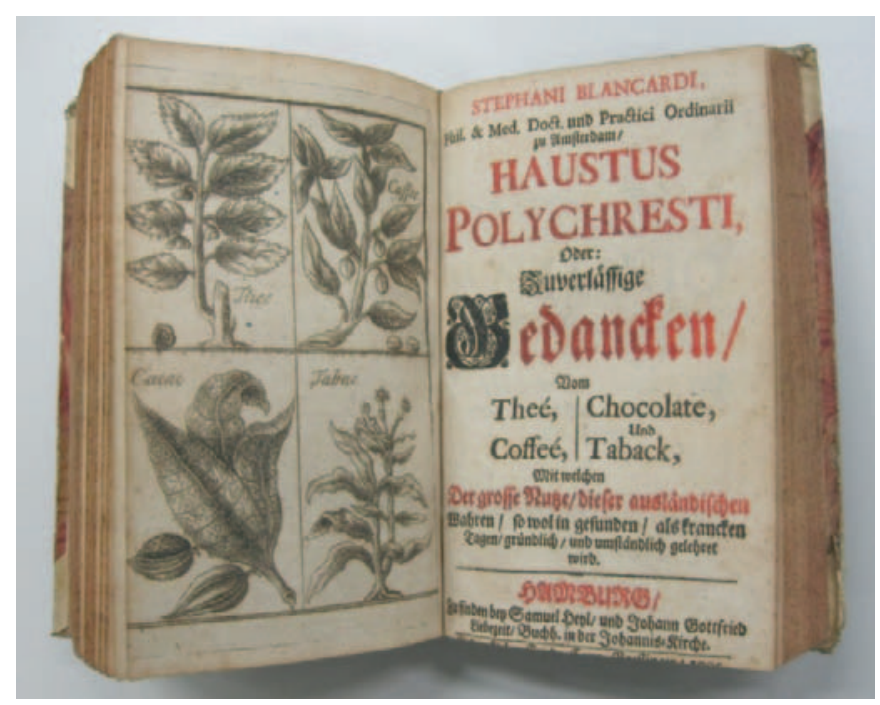

Abb. 3. Ramazzini Bernardino: «De Morbis artificum diatriba. accedunt Lucae Antonii Portii ... Dissertatio logica, Editio Secunda». Apud Guilielmum van de Water, Ultrajecti (Utrecht), 1703 (Foto: D-CHAB, ETH Zürich).

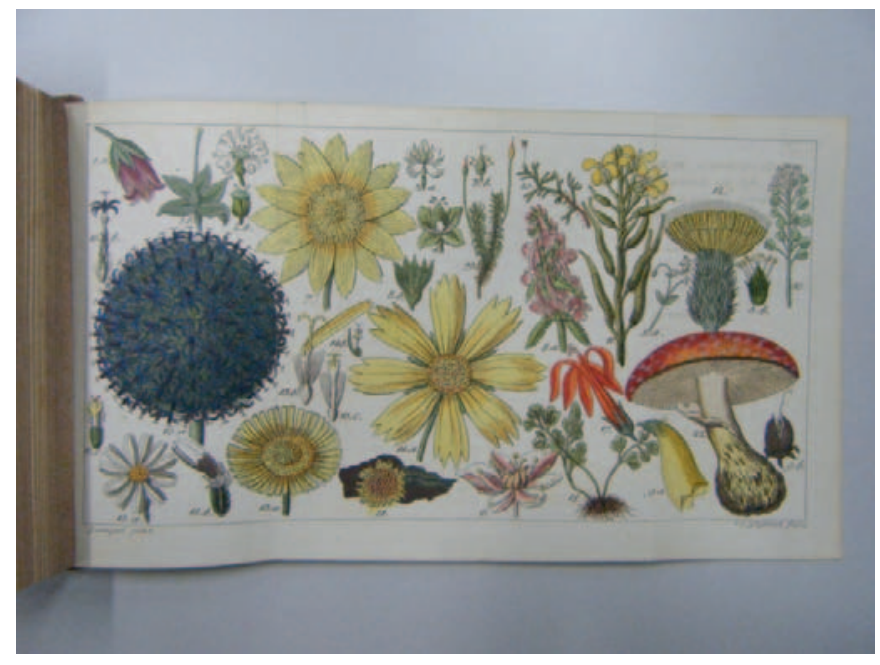

Abb. 4. Bei Ferdinand Oehmigke dem Aeltern: «Berlinisches Jahrbuch für die Pharmacie und für die damit verbundenen Wissenschaften auf das Jahr 1799 ...». Berlin (Foto: D-CHAB, ETH Zürich).
In vielen Büchern finden sich verzierte Darstellungen und bildliche Allegorien. Manche Anwendung von Heilpflanzen in den alten Kräuterbüchern scheint absonderlich; über so manche Darstellung von Einhorn oder Tod und Teufel kann gerätselt werden. Eindrucksvoll sind die vor Jahrhunderten akkurat gezeichneten wissenschaftlichen Apparaturen und die ästhetisch ansprechenden, künstlerisch hochstehenden Pflanzenzeichnungen (Abb. 1-4). Die Lektüre von Briefkorrespondenzen ausgewählter Buchautoren offenbarte nicht nur Forschungsvorlieben für bestimmte Pflanzen oder Tiere, sondern gab auch Einblicke in deren Familie, Lebensumstände, finanzielle Probleme, Zeitnot sowie den damals üblichen Austausch von Wissen und Pflanzenmaterial. Ebenso wurde erkennbarer, wer bevorzugt für begüterte Leser oder eher für die Allgemeinheit schrieb, wer «Bestseller» mit über 20 Auflagen verfasste oder heimlich von anderen abkopierte und ob Briefeschreiber miteinander befreundet waren oder in Konkurrenz zueinander standen.

\section{Viele Themen warten noch auf eine wissenschaftliche Bearbeitung}

Die Artikelreihe konnte nur einige wenige thematische Akzente setzen. Vieles könnte noch weiter analysiert, formal geordnet und unter verschiedenen historischen Gesichtspunkten untersucht werden. Etliche Bücher haben pharmakognostische, medizinische oder chemische Abhandlungen zum Thema, einige sind nur mit Text oder handschriftlichen Anmerkungen versehen, andere wiederum enthalten detaillierte, farbige Bilddarstellungen als Holzschnitt, Kupferstich oder später als Fotografie. Die von Hartwich erworbenen Pharmakopöen bzw. Dispensatorien und Apotheker-Taxen der Städte Amsterdam, Augsburg, Basel, Berlin, Halberstadt, Nürnberg, Schweinfurt, Stuttgart, Paris und Zürich könnten miteinander verglichen und dabei beispielsweise Wirkstoffgruppen, Rezepturen ausgewählter Mischungen, Verfügbarkeit bestimmter Arzneidrogen, Handel und Gesetzesgrundlagen der Länder, Regionen und Städte näher betrachtet werden.

Der vollständige Erhalt der Bibliothek und Sammlung Hartwichs macht die Arbeits- und Denkweise eines Pharmakognosie-Professors der Jahrhundertwende nachvollziehbar. Der Literaturschatz bestehend aus Kräuter- und Botanikbüchern, Traktaten, Dissertationen, Habilitationsschriften sowie Wochenzeitschriften und Jahrbüchern ermöglicht dem Leser einen Einblick in den jeweiligen Wissensstand der Pharmazie, Medizin und Chemie. Diesen Schatz gilt es in Zukunft nicht nur zu hüten und zu pflegen, sondern auch für weitere pharmazie- und medizinhistorische Forschungsarbeiten zu nutzen. 


\section{Weiterführende Internetseiten}

Departement Chemie und Angewandte Biowissenschaften $D-C H A B$

Damit die Bücherschätze in Zürich nicht so schnell in Vergessenheit geraten, wurden ihnen Schwerpunktthemen in Abendführungen, Vorträgen, Posterausstellungen und Diskussionsforen sowie ein kleines Buch gewidmet, einschliesslich einer Webseite mit ausgewählten Fotos (sortiert nach Erscheinungsdaten über fünf Jahrhunderte):

- www.chab.ethz.ch/publicrelations/collegium/

Diskussionsforen2015;

- www.chab.ethz.ch/publicrelations/collegium/Rezepturen_ Programm.pdf;
- www.chab.ethz.ch/publicrelations/collegium/Bucher;

- www.chab.ethz.ch/publicrelations/publikationen;

- www.chab.ethz.ch/museum/Sammlung/ Alte_Kraeuterbuecher.

Ausserdem:

- www.chab.ethz.ch/publicrelations;

- www.chab.ethz.ch/museum/index.

Pharmazie-Historisches Museum

- www.pharmaziemuseum.ch.

Papiermühle

- www.papiermuseum.ch.

\section{Literatur}

1 Eder R: Nachruf Prof. C. Hartwich. Schweizerische Apotheker-Zeitung 1917, Nr. 11.

2 Brauckmann B: Kräuterbücher (1): Sammelleidenschaft und Forschungseifer: Zwei Gelehrte aus verschiedenen Jahrhunderten auf den Spuren von Opium und Kaffee. Schweiz Z Ganzheitsmed 2014:26:105-107.

3 Brauckmann B: Kräuterbücher (2): Publikationskonflikte in der Renaissance: Leonhart Fuchs und Conrad Gessner im Streit um ihre Pflanzenwerke. Schweiz Z Ganzheitsmed 2014;26:168-171.
Kluge M: Kräuterbücher (3): Vom medizinischen Nachschlagewerk zum botanischen Lehrbuch: Die Entwicklung der Kräuterbuchliteratur. Schweiz Z Ganzheitsmed 2014;26: 223-226.

5 Brauckmann B: Kräuterbücher (4): Tulpen in den Prachtgärten der Kaufleute und Fürsten des 16. bis 18. Jahrhunderts: Erlesene Kostbarkeiten zu ruinösen Preisen. Schweiz Z Ganzheitsmed 2014;26:297-300.

6 Kluge M: Kräuterbücher (5): Einblicke in das Alchemielabor: Zwei bisher unbekannte Alchemistenhandschriften. Schweiz Z Ganzheitsmed 2014;26:350-353.
7 Brauckmann B: Kräuterbücher (6): Sammeln, Herausgeben und «Verzetteln» im 18. Jahrhundert: Brillante Pflanzenzeichner, geduldige Kupferstecher, rastlose Auftraggeber und eine liebende Ehefrau. Schweiz Z Ganzheitsmed 2015;27:36-40.

8 Brauckmann B: Kräuterbücher (7): Patienten behandeln, Reisen unternehmen, Pflanzen beobachten und Briefe schreiben: Mediziner und Botaniker in der Zeit des "Ancien Régime». Schweiz Z Ganzheitsmed 2015;27:95-99.

9 Kluge M: Kräuterbücher (8): Eine neue Sicht der Wissenschaft - wie das Mikroskop den Blick der Medizin veränderte. Schweiz Z Ganzheitsmed 2015;27:159-163. 